\title{
Trends of Postpartum Depression in Iran: A Systematic Review and Meta-Analysis
}

\author{
Yousef Veisani, ${ }^{1,2}$ Ali Delpisheh, ${ }^{2,3}$ Kourosh Sayehmiri, ${ }^{2,3}$ and Shahab Rezaeian ${ }^{4}$ \\ ${ }^{1}$ Student Research Committee, Ilam University of Medical Sciences, P.O. Box 69311-57793, Ilam, Iran \\ ${ }^{2}$ Department of Clinical Epidemiology, Faculty of Medicine, Ilam University of Medical Sciences, P.O. Box 69315-138, Ilam, Iran \\ ${ }^{3}$ Prevention of Psychosocial Injuries Research Centre, P.O. Box 69311-57793, Ilam, Iran \\ ${ }^{4}$ Department of Epidemiology, School of Health \& Nutrition, Shiraz University of Medical Sciences, P.O. Box 71348-14366, \\ Shiraz, Iran \\ Correspondence should be addressed to Kourosh Sayehmiri; sayehmiri@razi.tums.ac.ir
}

Received 31 March 2013; Revised 26 May 2013; Accepted 9 June 2013

Academic Editor: Klaus Ebmeier

Copyright (C) 2013 Yousef Veisani et al. This is an open access article distributed under the Creative Commons Attribution License, which permits unrestricted use, distribution, and reproduction in any medium, provided the original work is properly cited.

Background. Postpartum depression (PPD) is a serious mental health disorder affecting 13\% of women in developed communities. The present study reviews available epidemiological publications on PPD-related aspects in Iranian women to help policy makers and health workers to design preventative strategies and further researches. Materials and Methods. A systematic review was constructed based on the computerized literature valid database. The $95 \%$ confidence intervals were calculated by random effects models. Metaregression was introduced to explore and explain heterogeneity between studies. Data manipulation and statistical analyses were performed using Stata 11. Results. Overall, 41 studies met the inclusion criteria. The pooled prevalence of PPD in Iran was 25.3\% (95\% CI: 22.7\%-27.9\%). Amongst subgroups of unwanted delivery, illiterate, housewives, and having history of depression the prevalence was $43.4 \%$ (35.6-51.1), 31.6\% (18.1-45.0), 30.7\% (25.2-36.3), and 45.2\% (35.4-53.1), respectively. Conclusions. Interventions that would specifically target women with a prior history of depression, illiterates, housewives, or women with unwanted pregnancies could be helpful to decrease the prevalence of postpartum depression in Iran.

\section{Introduction}

Postpartum depression (PPD) affects almost $13 \%$ of women in developed high income communities [1] and may be even more common in developing countries [2,3]. According to the Diagnostic and Statistical Manual of Mental Disorders, Fourth Edition (DSM-IV), PPD is a major depression when symptoms have onset within 5 weeks of childbirth [4]. PPD presents with the same symptoms as for a major depressive episode occurring outside of the prenatal period, including core symptoms of depressed mood and/or loss of pleasure, together with additional symptoms, including changes in weight or sleep, fatigue or loss of energy, feelings of worthlessness or guilt, concentration difficulties, and suicidal ideation [4].

Majority of PPD researches in Iran have not utilized diagnostic assessments to identify cases. Alternatively, they have used the validated self-report depression screening instruments, such as the Edinburgh Postnatal Depression Scale (EPDS) [5]. Although this approach has been criticized, the EPDS has showed good sensitivity and specificity, particularly when used to detect both major and minor depressions [5].

In terms of etiology, PPD is a multifactorial disorder with biological, psychological, and sociological aspects interacting with woman's risk individually [6]. Sociological factors such as unwanted delivery, occupation, literacy, and history of depression have been more frequently reported throughout original researches and a meta-analysis [7]. However, they are not comprehensive as the present review is. Many of them drew incompatible or even contradictory conclusions, and the utilization of these statistics is therefore limited. The present study reviews available epidemiological publications on PPD-related aspects in Iranian women to help policy makers and health workers to design preventative strategies and further researches. 


\section{Methods and Materials}

2.1. Literature Search. Our search strategy, selection of publications, and the reporting of results for the review will be conducted in accordance with the PRISMA guidelines [8]. Literature on the postpartum depression among Iranian women was acquired through searching the Scientific Information Databases (SID), Global Medical Article Limberly (Medlib), Iranian Biomedical Journal (Iran Medex), and Iranian Journal Database (Magiran) as well as international databases including PubMed/Medline, Scopus, and ISI Web of Knowledge. The search strategy was limited to the Persian and/or English language papers published until Feb 2012. All publications with medical subject headings (MeSh) and keywords in title, abstract, and text for words including postpartum depression were investigated. Iranian scientific databases were searched only using the keyword "postpartum depression," as these databases do not distinguish synonyms from each other and do not allow sensitive search operation using linking terms such as "AND," "OR," or "NOT." Consequently, this single keyword search was the most practical option. The postpartum depression, depression, and Iran MeSh combined with the Selection and Quality Assessment of Articles operator “OR” versus “AND."

2.2. Selection and Quality Assessment of Articles. All identified papers were critically appraised independently by two reviewers. Disagreements between reviewers were resolved by consensus. Appraisal was guided by a checklist assessing clarity of aims and research questions. The inclusion criteria were as follows: (1) studies in the mentioned databases with full text, despite the language of original text; (2) having a standardized assessment of depression (either self-report or observer rated), using Edinburgh Postnatal Depression Scale (EPDS) and Beck depression inventory (BDI) instruments and study conducting of between 2 and 52 weeks postpartum were the main inclusion criteria. Exclusion criteria were (1) studies upon women overlapping time intervals of sample collection from the same origin; (2) inappropriate study design; (3) inadequate reporting of results.

2.3. Data Extraction. Data were extracted using a standardized and prepiloted data extraction form. Data extraction will be undertaken by the first reviewer and checked by a second reviewer although the process will be discussed and piloted by both reviewers. All identified papers will be critically appraised independently by both reviewers. Disagreements were resolved through discussion. Appraisal will be guided by a checklist assessing clarity of aims and research questions. Information was extracted from each included study (including author, title, year and setting of study, methods of sample selection, sample size, study type, age, and prevalence). Therefore, risk of bias as an "Inadequate Reporting" reduced. These data-abstraction forms were reviewed, and eligible papers were entered into the metaanalysis. Besides, as with all meta-analyses, this study has potential limitation of publication bias. Many of our data were extracted from studies written in Persian (language bias). However, we have confidence in our results since the included literature was published in non-Persian language, which should reduce publication bias to some extent.

2.4. Statistical Analysis. The random effects model was used for combining results of studies in meta-analysis. Variance for each study was calculated using the binomial distribution formula. The presence of heterogeneity was determined by the Der Simonian-Laird (DL) approach [9]. Significance level was $<0.1$, and $I^{2}$ statistic was used for estimates of inconsistency within the meta-analyses. The $I^{2}$ statistic estimates the percent of observed between-study variability due to heterogeneity rather than to chance and ranges from 0 to 100 percent (values of $25 \%, 50 \%$, and $75 \%$ were considered representing low, medium, and high heterogeneity resp.) [10]. A value of $0 \%$ indicates no observed heterogeneity whilst $100 \%$ indicates significant heterogeneity. For this review, we determined that $I^{2}$ values above 75 percent were indicative of significant heterogeneity warranting analysis with a random effect model as opposed to the fixed effects model to adjust for the observed variability. The $I^{2}$ is an estimate of the proportion of the total variation across studies that are beyond chance. In situations with high between-study heterogeneity, the use of random effects models is recommended as it produces study weights that primarily reflect the betweenstudy variation and thus provide close to equal weighting. Univariate and multivariate metaregression analyses were used to explore possible sources of heterogeneity among studies [11]. We analyzed sources of heterogeneity by subgroup and metaregression analysis using dichotomous and continuous variables. Univariate and multivariate approaches were employed to assess the causes of heterogeneity among the selected studies. Metaregression was used to show the trend of variation of prevalence during time. Egger test was conducted to examine potential publication bias. Egger's test can reveal a symmetric or asymmetric funnel plot. The latter indicates the existence of a significant publication bias or a systematic heterogeneity between studies. Data manipulation and statistical analyses were done using STATA software, version 11.2. $P$ values $<0.05$ were considered as statistically significant.

\section{Results}

According to the literature search strategies, 268 studies were identified, but 227 studies were excluded as they did not meet the inclusion criteria. There were 9 studies in English $[5,12-$ 19] and 32 studies in Persian [20-51] of the finally adopted 41 studies, and they were published between 1995 and 2012. The pooled sample sizes included 21907 women (Table 1 and Figure 1).

The heterogeneity between studies was $94.5 \%$ with an $I$ square $\left(I^{2}\right)$ statistic $(P \leq 0.001)$. The pooled prevalence of postpartum depression was 25\% (95\% CI: 22.7-27.9\%) (Figure 2). Based on the Edinburgh Postnatal Depression Scale (EPDS) and Beck depression inventory (BDI), the PPD prevalence in Iran was $24.3 \%$ (95\% CI: $21.0-27.7$ ) and $25.3 \%$ (95\% CI: 22.7-27.9), respectively. 
TABLE 1: Feature of studies among women with postpartum depression at different regions.

\begin{tabular}{|c|c|c|c|c|c|c|}
\hline Study number/author(s) & Place & $\begin{array}{c}\text { No. of } \\
\text { population }\end{array}$ & $\begin{array}{l}\text { Assessment } \\
\text { times }\end{array}$ & $\begin{array}{l}\text { Instrument } \\
\text { Assessment }\end{array}$ & Cut point & $\begin{array}{c}\text { Prevalence of } \\
\text { PPD (\%) }\end{array}$ \\
\hline (1) Kheirabadi et al. $[12,16]$ & Isfahan & 6627 & 2-12 weeks & EPDS & 13 & 30 \\
\hline (2) Khorramirad et al. [38] & Qom & 300 & $6-12$ weeks & EPDS & 13 & 23.7 \\
\hline (3) Hosseini Sazi et al. [34] & Gorgan & 180 & 2-14 weeks & BDI & 16 & 23.3 \\
\hline (4) Zangeneh et al. [51] & Kermanshah & 531 & $2-6$ weeks & EPDS & 13 & 40.7 \\
\hline (5) Azimi Lolati et al. [24] & Sari & 442 & 6-8 weeks & EPDS & 13 & 22 \\
\hline (6) Sehhatie Shafaei et al. [49] & Tabriz & 600 & $10-20$ weeks & EPDS & 12 & 34.7 \\
\hline (7) Shobeiri et al. [50] & Hamadan & 400 & 2-8 weeks & BDI & 16 & 32 \\
\hline (8) Khodadadi et al. [36] & Rasht & 350 & $6-8$ weeks & EPDS & 13 & 16 \\
\hline (9) Hosseini et al. [33] & Kermanshah & 330 & 4-14 weeks & BDI & 16 & 24.8 \\
\hline (10) Ghaffari et al. [31] & Ramsar & 100 & $6-16$ weeks & GHQ 28 & 22 & 36.6 \\
\hline (11) Foruzandeh and Dashtebozorgi [30] & Shahrekord & 300 & $6-10$ weeks & BDI & 16 & 21.3 \\
\hline (12) Bahrami et al. [26] & Dezful & 140 & $6-10$ weeks & EPDS & 13 & 31.4 \\
\hline (13) Dolatian et al. [28] & Tehran & 285 & $2-6$ weeks & EPDS & 12 & 20.3 \\
\hline (14) Ajh et al. [22] & Astaneh & 440 & 2-4 weeks & BDI & 16 & 18.6 \\
\hline (15) Khooshemehry et al. [37] & Tehran & 250 & $6-52$ weeks & BDI & 16 & 30 \\
\hline (16) Tabrizi et al. [52] & Hamadan & 144 & 2-12 weeks & BDI & 16 & 16.8 \\
\hline (17) Barekatain et al. [27] & Isfahan & 1898 & $6-8$ weeks & EPDS & 13 & 24.4 \\
\hline (18) Rahmani et al. [45] & Tabriz & 560 & $8-52$ weeks & EPDS & 12 & 32 \\
\hline (19) Jafarpour et al. [35] & Kermanshah & 975 & 13-26 weeks & EPDS & 12 & 17.5 \\
\hline (20) Mousavi et al. [42] & Kashan & 204 & 9-13 weeks & BDI & 13 & 34.7 \\
\hline (21) Bagherzadeh et al. [25] & Bushehr & 400 & 2-12 weeks & EPDS & 15 & 15.5 \\
\hline (22) Kiani et al. [39] & Astara & 105 & $2-4$ weeks & EPDS & 12 & 25 \\
\hline (23) Kamranpour and Shakiba [20] & Rasht & 310 & $2-8$ weeks & EPDS & 12 & 6.4 \\
\hline (24) Dolatian et al. $[28,29]$ & Marivan & 204 & $2-6$ weeks & EPDS & 10 & 34.2 \\
\hline (25) Salary et al. [47] & Mashhad & 60 & $2-4$ weeks & EPDS & 10 & 9.9 \\
\hline (26) Lashkaripour et al. [40] & Zahedan & 300 & 4-18 weeks & BDI & 15 & 33.7 \\
\hline (27) Nikpour et al. [43] & Amol & 420 & $2-8$ weeks & EPDS & 12 & 10.3 \\
\hline (28) Asadi Sadeghi Azar et al. [13] & Zabol & 408 & $2-8$ weeks & BDI & 16 & 40.4 \\
\hline (29) Aghapoor and Mohammadi [21] & Tabriz & 300 & $6-12$ weeks & $\mathrm{BDI}$ & 16 & 28 \\
\hline (30) Ghaffari Nejad et al. [32] & Kerman & 400 & $2-8$ weeks & BDI & 16 & 31.1 \\
\hline (31) Akbari et al. [53] & Hamadan & 159 & $4-8$ weeks & EPDS & 13 & 26.4 \\
\hline (32) Salehi [48] & Hormozgan & 164 & $2-8$ weeks & EPDS & 13 & 14.9 \\
\hline (33) Sadr et al. [46] & Tehran & 300 & $2-8$ weeks & EPDS & 13 & 23.7 \\
\hline (34) Taavoni et al. [14] & Tehran & 597 & 4-12 weeks & EPDS & 13 & 22.6 \\
\hline (35) Alipour et al. [15] & Qom & 160 & 4-12 weeks & EPDS & 13 & 25 \\
\hline (36) Montazeri et al. [5] & Isfahan & 100 & 12-14 wWeks & EPDS & 13 & 22 \\
\hline (37) Kheirabadi et al. $[12,16]$ & Isfahan & 1291 & 6-8 weeks & EPDS & 13 & 26.3 \\
\hline (38) Iranfar et al. [17] & Kermanshah & 163 & $2-8$ weeks & BDI & 10 & 48.7 \\
\hline (39) Abbaszadeh et al. [18] & Kerman & 400 & $8-28$ weeks & EPDS & 13 & 45.8 \\
\hline (40) Tashakori et al. [19] & Ahvaz & 210 & $8-28$ weeks & EPDS & 12 & 21.4 \\
\hline (41) Akbarzadeh et al. [23] & Shiraz & 400 & 2-8 weeks & BDI & 16 & 21.1 \\
\hline
\end{tabular}

Amongst subgroups of unwanted delivery, illiterate, housewives, and having history of depression, the prevalence was $43.4 \%$ (35.6-51.1), 31.6\% (18.1-45.0), 30.7\% (25.2-36.3), and $45.2 \%$ (35.4-53.1), respectively (Table 2 ). A significant geographic difference in pooled PPD was observed.

The lowest PPD rate was observed in central, and the highest rate was observed in west and south-eastern border areas of Iran (Figure 3).

The metaregression of the prevalence PPD for each study on the interval sample size showed a negative and no statistically significant relationship $(\beta=-0.0003$, s.e. $(\beta)=$ $0.0002, P=0.995$ ) and no statistically significant change in prevalence over the time $(\beta=-0.4539$, s.e. $(\beta)=0.025$, $P=0.536$ ) (Figure 4 ). Since 1995, the PPD rates showed an increasing trend (Figure 5).

\section{Discussion}

Comprehensiveness of available information and a large sample size made the present study representative. There have been recent systematic reviews of studies dealing with risk factors of PPD in Iranian women [7], but the present study aimed to determine prevalence of PPD by a systematic review and meta-analysis method. The psychometric properties of 


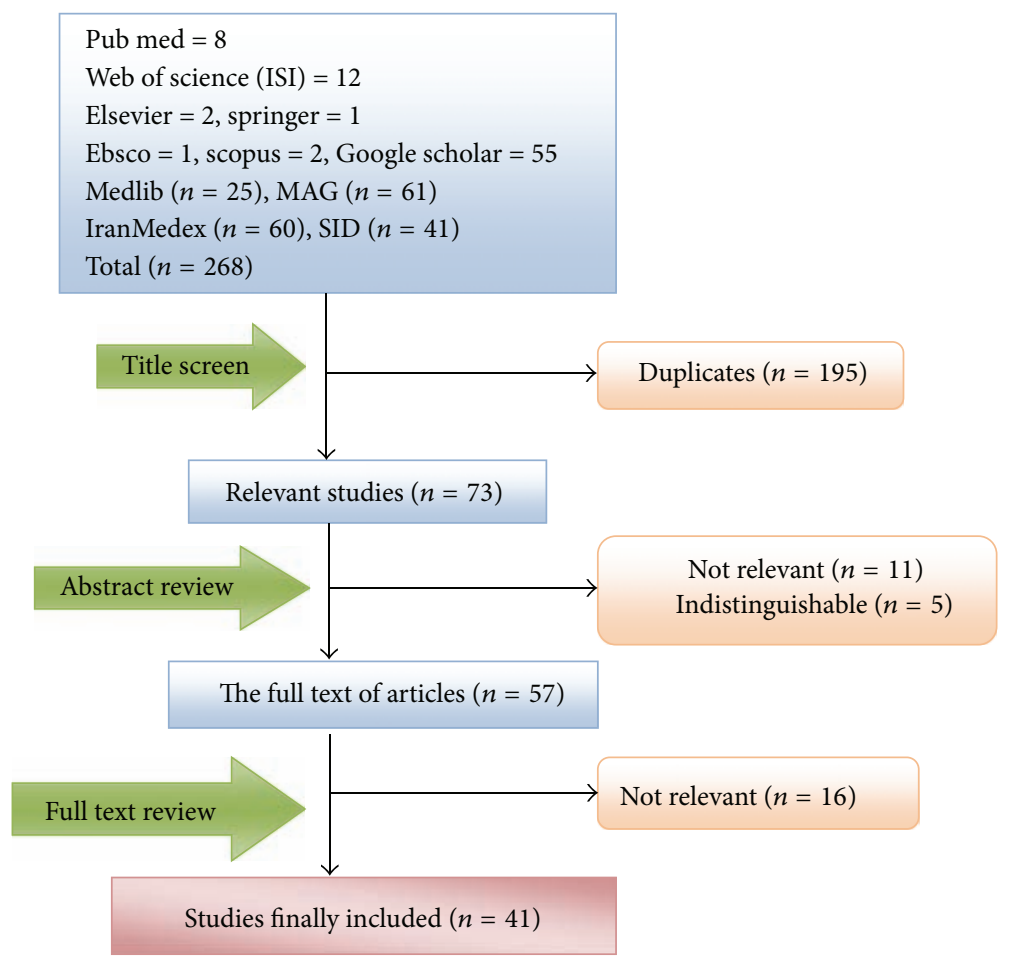

FIGURE 1: Results of the systematic literature search.

two main screening tools such as the Edinburgh Postnatal Depression Scale (EPDS) and Beck depression inventory (BDI) have been repeatedly assessed across the world.

The present study showed that the pooled prevalence of PPD among Iranian women was $25.3 \%$, which is similar to the finding of a recent meta-analysis reported by Paulson and colleagues (25.6\%) [54]. But it was not consistent with another meta-analysis of $15.6 \%$ [55]. This difference may be due to the assessment tools, geographic, and cut-off point differences. Prior studies that have noted the importance of recurrent PPD in women with a prior episode of postpartum affective psychosis may be at risk for recurrence postpartum $50-70 \%$ [56].

A significant geographic difference of the prevalence of PPD was also observed. Compared with other regions, west and south-eastern borders of Iran had relatively higher prevalence rates, accounting for $48.7 \%$ and $40.4 \%$, respectively. Meanwhile, the lowest prevalence rate was found in north of Iran of 6.4\%. Notably, during the period through 1995 and 2012, the prevalence rates were commonly at high level among different regions of Iran especially in border regions; the rates even reached nearly 50\%. Possible reasons for this lack of reduction may include backwardness of border province to the central provinces, poor economic conditions, lack of program education in the sensitive groups, or limited sampling.

An extensive list of characteristics of PPD was examined among a diverse and representative sample of Iranian women. Conducting this study with a large sample size increased the statistical power. Sociological factors in Iran such as unwanted delivery as a result of the lack of family planning, illiterate result of gender discrimination, and carrier because most women are housewives have been more studied. The results of this study indicate that prevalence of PPD in illiterate women, unwanted delivery, and housewives women with a prior history of depression was $31.6 \%, 43.4 \%, 30.7 \%, 45.2 \%$, respectively. Rates of relapse are particularly high in women with a prior history of depression with estimates ranging from $25 \%-50 \%$ [57]. Interventions that would specifically target women with a prior history of depression, illiterate women, housewives mothers, or women with unwanted pregnancy may help to decrease the prevalence of PPD among this population.

The strengths of this review include the large number of samples included and therefore the ability to examine prevalence in clinically relevant subgroups with some degree of precision. The $I^{2}$ is an estimate of the proportion of the total variation across studies that is beyond chance. In situations with high between-study heterogeneity, the use of random effects models is recommended as it produces study weights that primarily reflect the between-study variation and thus provides close to equal weighting. Univariate and multivariate metaregression analyses were used to explore possible sources of heterogeneity among studies [11]. Furthermore, we have examined heterogeneity using subgroup analyses and metaregression, which allowed us to investigate dichotomous and continuous variables such as age, sample size, and the date when the study was conducted. According to the metaregression analysis, none of the data were not statistically significant with changes of PPD prevalence. The high levels of heterogeneity between the studies are to be expected as the studies were conducted in different samples, 
TABLE 2: Prevalence of postpartum depression among subgroups.

\begin{tabular}{|c|c|c|c|c|c|c|}
\hline \multirow{2}{*}{ Variables } & \multirow{2}{*}{ No. of studies } & \multirow{2}{*}{ No. of patients } & \multirow{2}{*}{ Prevalence \% (95\% CI) } & \multicolumn{2}{|c|}{ Heterogeneity } & \multirow{2}{*}{ Model } \\
\hline & & & & $I^{2}$ & $P$ value & \\
\hline Housewife & 8 & 10756 & $30.7(25.2-36.3)$ & $96.4 \%$ & 0.000 & REM $^{*}$ \\
\hline Employee & 8 & 10756 & $29.3(21.8-36.8)$ & $96.4 \%$ & 0.000 & REM \\
\hline History of depression & 8 & 10787 & $45.2(35.4-53.1)$ & $97.9 \%$ & 0.000 & REM \\
\hline No history of depression & 8 & 10787 & $27.3(21.1-33.5)$ & $97.3 \%$ & 0.000 & REM \\
\hline Unwanted delivery & 11 & 11702 & $43.4(35.6-51.1)$ & $98.3 \%$ & 0.000 & REM \\
\hline Desired delivery & 11 & 11702 & $29.0(23.5-34.6)$ & $97.1 \%$ & 0.000 & REM \\
\hline Illiterate & 4 & 7688 & $31.6(18.1-45.0)$ & $98.3 \%$ & 0.000 & REM \\
\hline literate & 4 & 7688 & $41.1(25.1-57.1)$ & $98.7 \%$ & 0.000 & REM \\
\hline Pooled & 41 & 21907 & $25.3(22.7-27.9)$ & $94.8 \%$ & 0.000 & REM \\
\hline
\end{tabular}

${ }^{*}$ Random effects model.

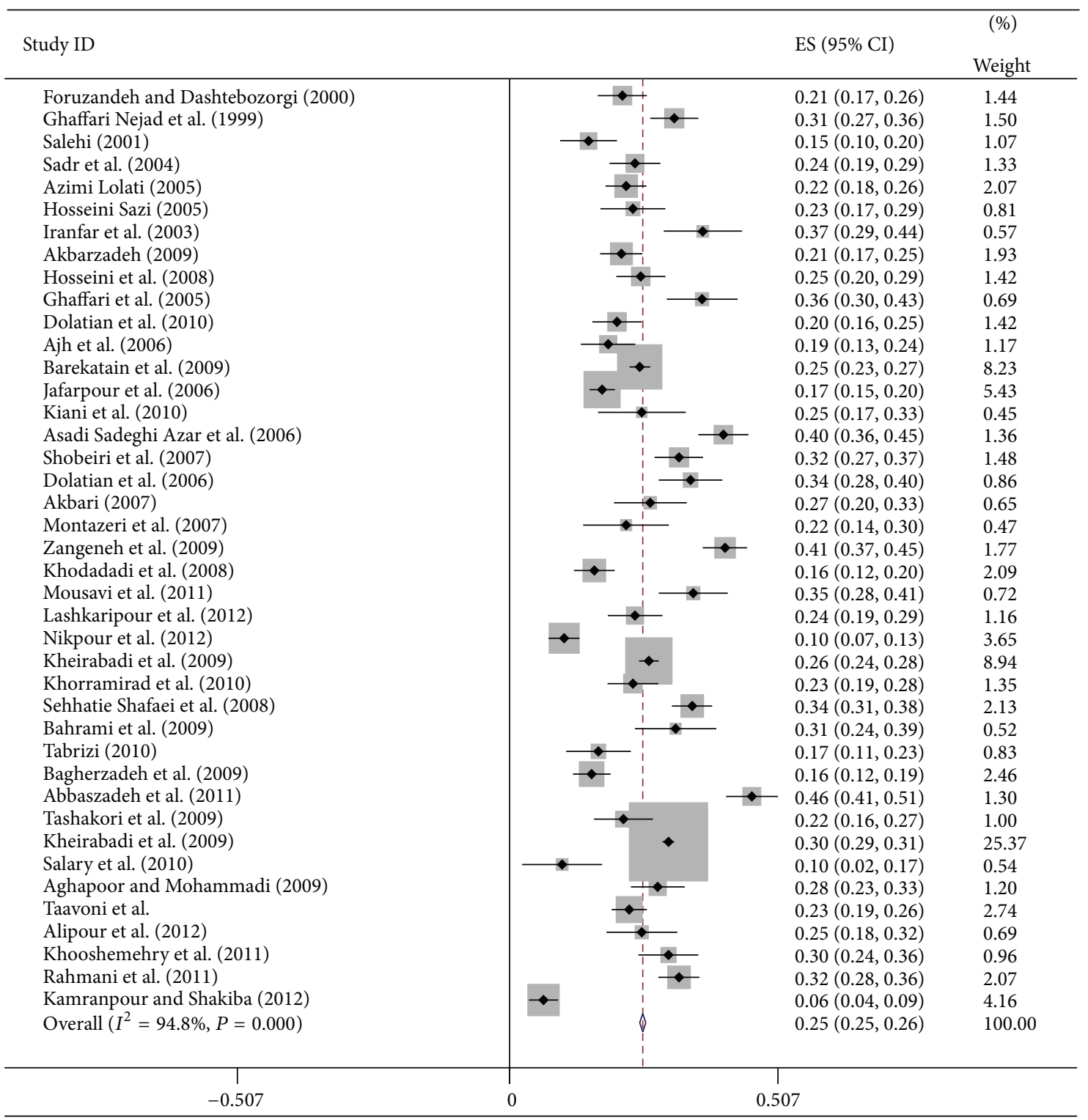

FIGURE 2: Forest plots for random effects meta-analyses. CI indicates confidence interval. 

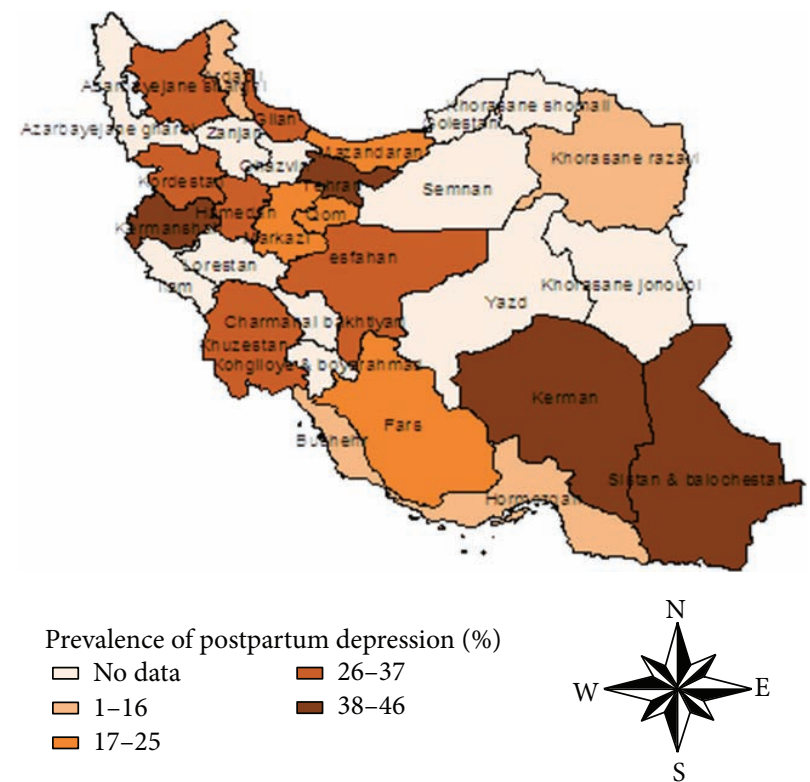

FIGURE 3: Regional distribution of pooled prevalence of postpartum depression in Iran.

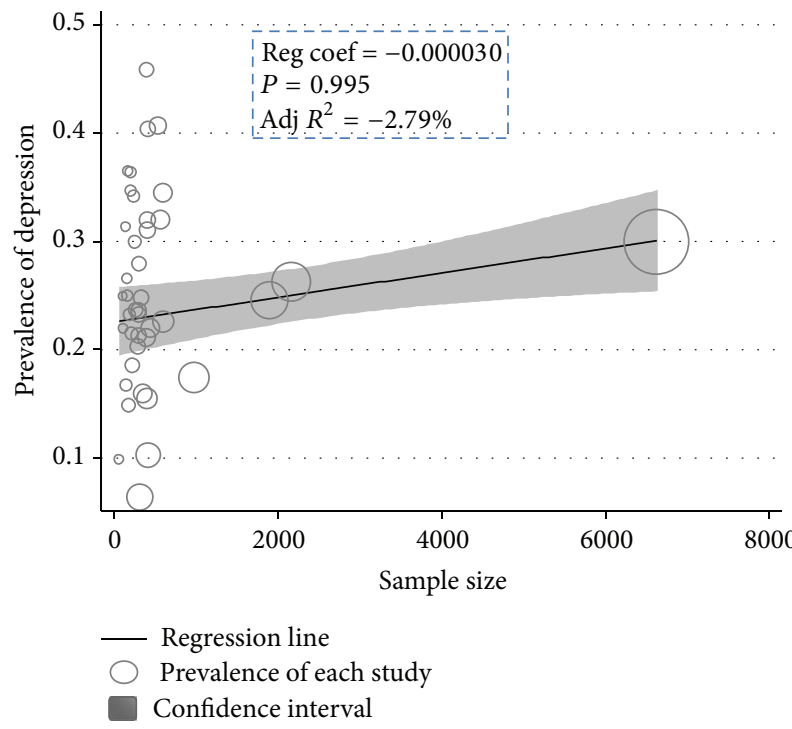

(a)

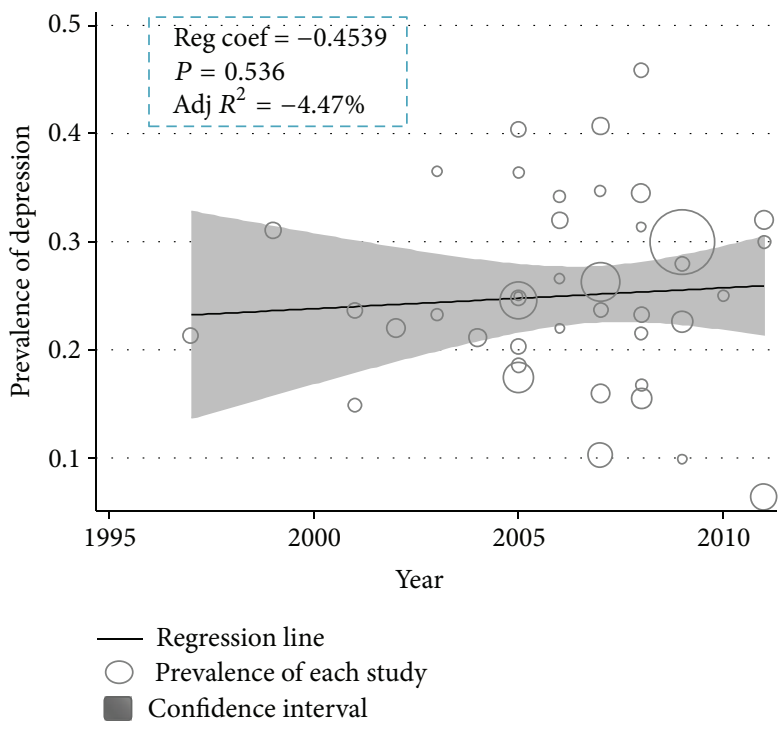

(b)

FIGURE 4: Metaregression plots of change in PPD according to changes in continuous study moderator's year and sample size.

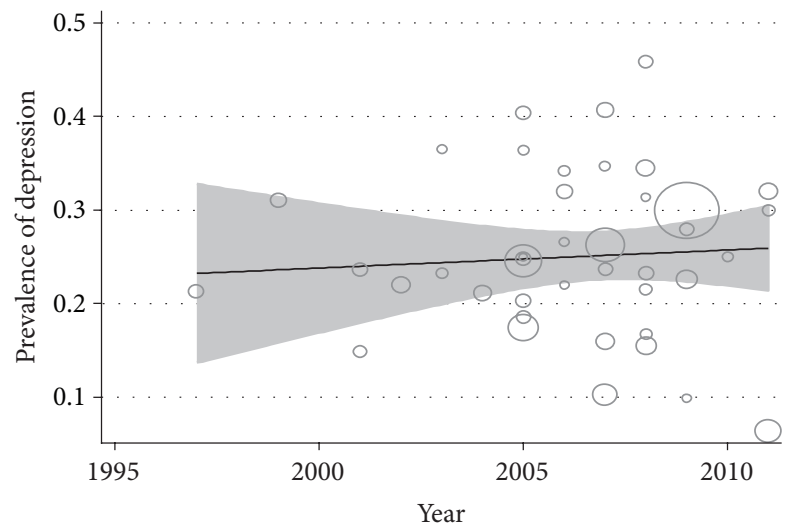

FIGURE 5: Prevalence of postpartum depression among Iranian women at different study periods. 
and this may simply reflect real differences in prevalence over time and by region. Particular reasons for this difference in women are unclear. Our approach to this was to identify causes of heterogeneity, and two possible explanations were assessed. Possibilities include that in the diagnostic systems, fatigability is included in the core criteria for depression. In addition, it may be that the distinction between minor and major forms of depression is more important in women as the overlap between sadness and clinical depression is more difficult to determine.

There are some limitations in the present study which need to be addressed. First of all, more studies were observational and patients were not randomly chosen. Therefore, selection bias and confounding seem inevitable. Secondly, much of our data were extracted from the internal databases in Iran. Thirdly, our ability to assess study quality was limited by the fact that many studies failed to offer detailed information on selected subjects or valid data on important factors, and in the end timing of the administration of the EPDS and information bias may be present due to the selfreport nature, and eventually in this review, there may be other explanations for the heterogeneity that we did not test, such as comorbidity with other mental disorders, but systematic data on this were lacking.

\section{References}

[1] B. N. Gaynes, N. Gavin, S. Meltzer-Brody et al., "Perinatal depression: prevalence, screening accuracy, and screening outcomes," Evidence Report/Technology Assessment, no. 119, pp. 1-8, 2005.

[2] H. M. Ahmed, S. K. Alalaf, and N. G. Al-Tawil, "Screening for postpartum depression using Kurdish version of Edinburgh postnatal depression scale," Archives of Gynecology and Obstetrics, vol. 285, no. 5, pp. 1249-1255, 2011.

[3] F. H. Al Dallal and I. N. Grant, "Postnatal depression among Bahraini women: prevalence of symptoms and psychosocial risk factors," Eastern Mediterranean Health Journal, vol. 18, no. 5, pp. 439-445, 2012.

[4] American Psychiatric Association, Diagnostic and Statistical Manual of Mental Disorders, American Psychiatric Press, Washington, DC, USA, 1994.

[5] A. Montazeri, B. Torkan, and S. Omidvari, "The edinburgh postnatal depression scale (EPDS): translation and validation study of the Iranian version," BMC Psychiatry, vol. 7, no. 11, pp. $1-6,2007$.

[6] P. Klainin and D. G. Arthur, "Postpartum depression in Asian cultures: a literature review," International Journal of Nursing Studies, vol. 46, no. 10, pp. 1355-1373, 2009.

[7] P. Bahadoran, S. S. Foroushani, S. Ehsanpour, and A. Abedi, "A meta-analysis on studies about obstetric risk factors of postpartum depression in Iran within 1995-2005," Iranian Journal of Nursing and Midwifery Research, vol. 13, no. 4, pp. 129-133, 2008.

[8] P. Grossman, L. Niemann, S. Schmidt, and H. Walach, "Mindfulness-based stress reduction and health benefits: a meta-analysis," Journal of Psychosomatic Research, vol. 57, no. 1, pp. 35-43, 2004.

[9] R. DerSimonian and N. Laird, "Meta-analysis in clinical trials," Controlled Clinical Trials, vol. 7, no. 3, pp. 177-188, 1986.
[10] A. E. Ades, G. Lu, and J. P. T. Higgins, "The interpretation of random-effects meta-analysis in decision models," Medical Decision Making, vol. 25, no. 6, pp. 646-654, 2005.

[11] M. Borenstein, L. V. Hedges, J. P. T. Higgins, and H. R. Rothstein, Introduction to Meta-Analysis, John Wiley \& Sons, 2009.

[12] G.-R. Kheirabadi, M.-R. Maracy, M. Barekatain et al., "Risk factors of postpartum depression in rural areas of Isfahan Province, Iran," Archives of Iranian Medicine, vol. 12, no. 5, pp. 461-467, 2009.

[13] I. Asadi Sadeghi Azar, Z. Hashemi, and F. Forghani, "Postpartum depression and its correlates among women living in zabol (Iran)," Iranian Journal of Psychiatry, vol. 1, no. 4, pp. 140-147, 2006.

[14] S. Taavoni, P. Zarea, and H. Haghani, "P-532-postpartum depression: related factors in clients, whom had been visited in clinics of tehran university of medical sciences," European Psychiatry, vol. 27, Supplement 1, article 1.

[15] Z. Alipour, M. Lamyian, and E. Hajizadeh, "Anxiety and fear of childbirth as predictors of postnatal depression in nulliparous women," Women and Birth, vol. 25, no. 3, pp. e37-e43, 2012.

[16] G. R. Kheirabadia and M. R. Maracy, "Perinatal depression in a cohort study on Iranian women," Journal of Research in Medical Sciences, vol. 15, no. 1, pp. 41-49, 2010.

[17] S. Iranfar, J. Shakeri, M. Ranjbar, P. NazhadJafar, and M. Razaie, "Is unintended pregnancy a risk factor for depression in Iranian women?" Eastern Mediterranean Health Journal, vol. 11, no. 4, pp. 618-624, 2005.

[18] A. Abbaszadeh, F. P. Kermani, H. Safizadeh, and N. Nakhee, "Violence during pregnancy and postpartum depression," Pakistan Journal of Medical Sciences, vol. 27, no. 1, pp. 177-181, 2011.

[19] A. Tashakori, A. Shanesaz, and A. Rezapour, "Assessment of some potential risk factors of postpartum depression," Pakistan Journal of Medical Sciences, vol. 25, no. 2, pp. 261-264, 2009.

[20] S. B. Kamranpour and M. Shakiba, "Cesarean section and post partum depression," Iranian Journal of Obstetrics, Gynecology and Infertility, vol. 15, no. 1, part 41, pp. 56-62, 2012.

[21] M. Aghapoor and A. Mohammadi, "Comparing postpartum depression of working women and housewives and its relationship with social support and marital adjustment," Women \& Study of Family, vol. 1, no. 4, pp. 9-32, 2009.

[22] N. Ajh, M. Unesian, A. Fili, and A. Abasi Motejaded, "The study of supportive activities during pregnancy on postpartum depression," Journal of Nursing and Midwifery, vol. 12, no. 3, pp. 73-80, 2006

[23] M. Akbarzadeh, F. Sharif, N. Zare, and F. Ghodrati, "Prevalence of symptoms post-partum anxiety and baby blues and factors effective uponit in women with high risk pregnancies," Journal of Family Research, vol. 5, no. 1, pp. 57-71, 2009.

[24] H. Azimi Lolati, M. M. Danesh, S. H. Hosseini, A. R. Khalilian, and M. Zarghami, "Postpartum depression in clients at health care centers in Sari," Iranian Journal of Psychiatry and Psychology, vol. 111, no. 40, pp. 31-42, 2005.

[25] R. Bagherzadeh, N. Zahmatkeshan, N. Moatamed, R. Khorramroudi, and M. Ganjoo, "Prevalence of maternal blues, postpartum depression and their correlation with premenstrual syndrome in women refferred to health centers affiliated to bushehr university of medical sciences," Iranian Journal of Obstetric, Gynecology and Infertility, vol. 12, no. 3, pp. 9-15, 2009.

[26] N. Bahrami, M. Araban, and S. Bahrami, "The Impact of antenatal education on postpartum," Journal of Hormozgan University of Medical Sciences, vol. 13, no. 14, pp. 277-283, 2009. 
[27] M. Barekatain, M. Tavakkoli, G. Kheirabadi, and M. R. Maracy, "The relationship between life-time prevalence of bipolar spectrum disorders and incidence of postnatal depression," Iranian Journal of Psychiatry and Clinical Psychology, vol. 15, no. 2, pp. 183-192, 2009.

[28] M. Dolatian, K. Hesami, J. Shams, and H. A. Majd, "Relationship between violence during pregnancy and postpartum depression," Iranian Red Crescent Medical Journal, vol. 12, no. 4, pp. 377-383, 2010.

[29] M. Dolatian, P. Maziar, H. Alavi-Majd, and M. Yazdjerdi, "The relationship between mode of delivery and postpartum depression," Journal of Reproduction and Infertility, vol. 7, no. 328, pp. 260-268, 2006.

[30] N. Foruzandeh and B. Dashtebozorgi, "Prevalence and predisposing factors of post-partum depression among women referred to the health care centers of Shahrekord, 1996," Journal of Shahrekord University of Medical Sciences, vol. 2, no. 1, pp. 4351, 2000.

[31] F. Ghaffari, T. Pourghaznain, and R. Mazlom, "A mental health of women and their husbands during pregnancy and postpatrum in health center of ramsar," Journal of Gorgan University of Medical Sciences, vol. 5, no. 5, pp. 74-80, 2005.

[32] A. R. Ghaffari Nejad, F. Khobyari, and F. F. P, "Prevalence of postpartum depression in Kerman," Quarterly Journal of Mentality \& Behavior, vol. 5, article 1, 1999.

[33] H. Hosseini, A. H. Naghibi, and M. Khademloo, "Postpartum depression and relaship with some related factors," Journal of Babol University of Medical Sciences, vol. 10, no. 2, pp. 76-81, 2008.

[34] F. Hosseini Sazi, A. Poor Reza, M. Hosseini, and D. Shojaei Zadeh, "Depression during pregnancy," Journal of Gorgan University of Medical Sciences, vol. 7, no. 1, pp. 60-65, 2005.

[35] M. Jafarpour, M. Esfandyari, Mokhtarsahi sh, and F. Hoseini, "The effect of stressful life events on postpartum depression," University of Medical Sciences, Kermanshah, vol. 10, no. 4, pp. 320-331, 2006.

[36] N. Khodadadi, H. Mahmoudi, and N. Mir hagh jo, "Postpartum depression relationship with some psychosocial effects in mothers," Journal of Ardabil University of Medical Sciences, vol. 8, no. 2, pp. 142-148, 2008.

[37] G. Khooshemehry, A. Shariati Feizabady, V. Naserkhaki, and V. Naserkhak, "Prevalence of postpartum depression and the factors that decides in clinics in the North of Tehran," Journal of Nursing and Midwifery, vol. 19, no. 1, pp. 59-64, 2011.

[38] A. Khorramirad, M. Mousavi Lotfi, and A. Shoori Bidgol, "Prevalence of postpartum depression and related factors in qom," Pejohandeh, vol. 15, no. 2, part 74, pp. 62-66, 2010.

[39] F. Kiani, T. Khadivzadeh, M. R. Sargolzaee, and H. Behnam, "Relationship between marital satisfaction during Pregnancy and Postpartum Depression (PPD)," Iranian Journal of Obstetrics, Gynecology and Infertility, vol. 13, no. 5, pp. 37-44, 2010.

[40] K. Lashkaripour, N. Bakhshani, S. Hokmabadi, A. R. SajjadiAmeneh, and A. Safarzadeh Sarasiyab, "Postpartum depression and related factors: a 4.5 months study," Journal of Fundamentals of Mental Health, vol. 134, no. 52, pp. 404-412, 2012.

[41] M. J. Modabernia, H. Shodjaei tehrani, and S. Heydari Nezhad, "Survey the frequency of depression in the last third months of pregnancy," Journal of Guilan University of Medical Sciences, vol. 18, no. 71, pp. 19-25, 2009.

[42] G. A. Mousavi, S. Sabahi Bidgoli, A. Omidi et al., "Prevalence of postpartum depression and its relation to some psychosocial factors in mothers referred to health centers of Kashan during 2007-8," Faiz, vol. 15, no. 3, pp. 247-253, 2011.

[43] M. Nikpour, Z. Abedian, N. Mokhber, S. Ebrahimzadeh, and S. Khani, "Relationship between delivery method and postpartum depression," Journal of Fundamentals of Mental Health, vol. 14, no. 1, part 53, pp. 46-53, 2012.

[44] F. Pazande, J. Tominas, F. Valai, and N. Afshar, "Prevalence of depression and its associated factors in women's centers martyr Beheshti University of Medical Sciences, 1999," Faiz, no. 28, pp. 66-70, 2002.

[45] F. Rahmani, N. Seyedfatemi, M. Asadollahi, and A. Seyedrasooli, "Predisposing factors of postpartum depression," Iran Journal of Nursing, vol. 24, no. 72, pp. 78-87, 2011.

[46] S. S. Sadr, M. Dowlatian, and Z. Behboudi Moghadam, "Prevalence of postpartum depression and factors affecting it in Tehran," Journal of Medical Council of IRI, vol. 22, no. 3, pp. 189193, 2004.

[47] P. Salary, E. Banafshe, P. Hebrani, and J. Jabbari Nooghabi, "On the relationship between maternal fatigue and postpartum depression," Journal of Fundamentals of Mental Health, vol. 114, no. 14, pp. 302-311, 2010.

[48] L. Salehi, "Postpartum depression prevalence in primiparous and multiparous women," Journal of Hormozgan University of Medical Sciences, vol. 5, no. 2, pp. 22-25, 2001.

[49] F. Sehhatie Shafaei, F. Ranjbar Koochaksariie, M. Ghojazadeh, and Zh. Mohamadrezaei, "Study of relationship between some predisposing factors and postpartum depression," Journal of Ardabil University of Medical Sciences, vol. 81, no. 27, pp. 54-61, 2008.

[50] F. Shobeiri, A. Farhadi Nasab, and M. Nazar, "Detecting postpartum depression in referents to medical and health centers in hamadan city," Scientific Journal of Hamadan University of Medical Sciences and Health Services, vol. 14, no. 3, pp. 24-28, 2007.

[51] M. Zangeneh, N. S. Alizadeh, M. Kaamrvamanesh, M. Rezaie, and S. Pormehr, "Postpartum depression and its relation to baby gender and unplanned pregnancy," Scientific Journal of Kurdistan University of Medical Sciences, vol. 14, no. 2, pp. 65-71, 2009.

[52] M. Tabrizi and K. H. Lorestani, "Eclectic effectiveness of interventions to reduce depression in pregnancy and prevention of Postpartum Depression," Thought and Behavior in Clinical Psychology, vol. 5, no. 17, pp. 19-26, 2010.

[53] S. Amir Ali Akbari, M. Toghiri, N. Karimian, and H. AlaviMajd, "Association between anemia during pregnancy and postpartum depression," Journal of Nursing and Midwifery, vol. 16, no. 54, pp. 19-26, 2007.

[54] J. F. Paulson and S. D. Bazemore, "Prenatal and postpartum depression in fathers and its association with maternal depression: a meta-analysis," Journal of the American Medical Association, vol. 303, no. 19, pp. 1961-1969, 2010.

[55] J. Fisher, M. C. de Mello, V. Patel et al., "Prevalence and determinants of common perinatal mental disorders in women in low-and lower-middle-income countries: a systematic review," Bulletin of the World Health Organization, vol. 90, no. 2, pp. 139149, 2012.

[56] L. S. Cohen, "Pharmacologic treatment of depression in women: PMS, pregnancy, and the postpartum period," Depression and Anxiety, vol. 8, no. 1, pp. 18-26, 1998.

[57] K. L. Wisner, J. M. Perel, and R. L. Findling, "Antidepressant treatment during breast-feeding," American Journal of Psychiatry, vol. 153, no. 9, pp. 1132-1137, 1996. 


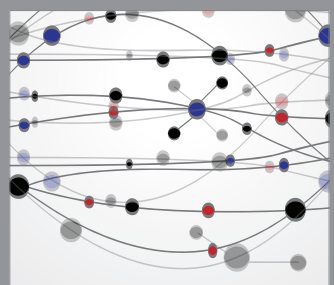

The Scientific World Journal
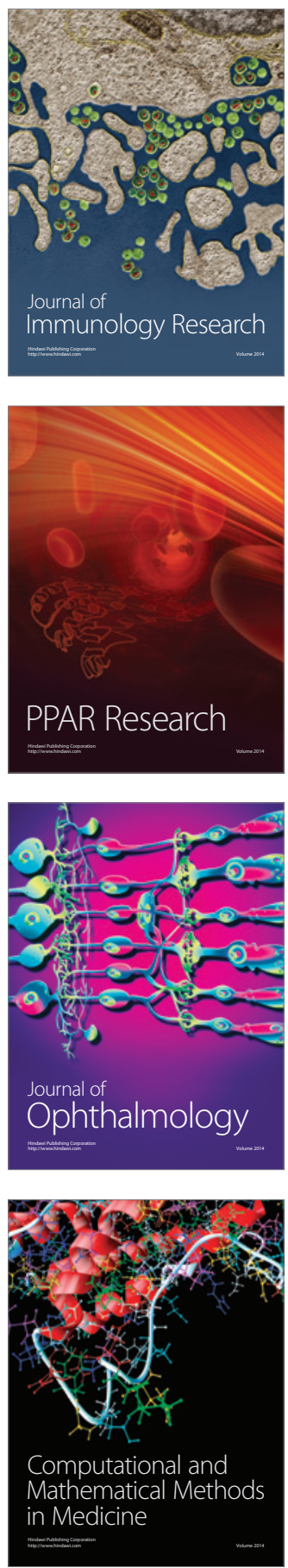

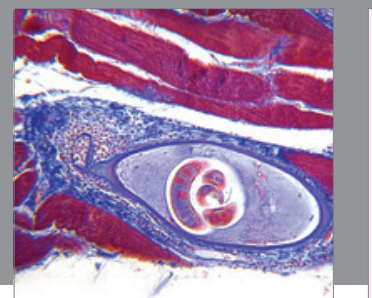

Gastroenterology

Research and Practice
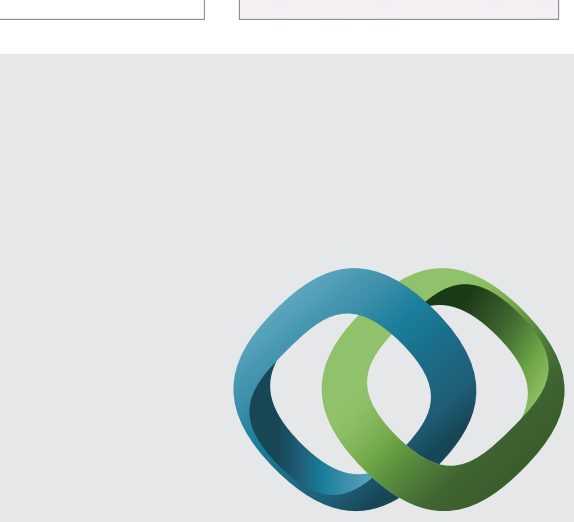

\section{Hindawi}

Submit your manuscripts at

http://www.hindawi.com
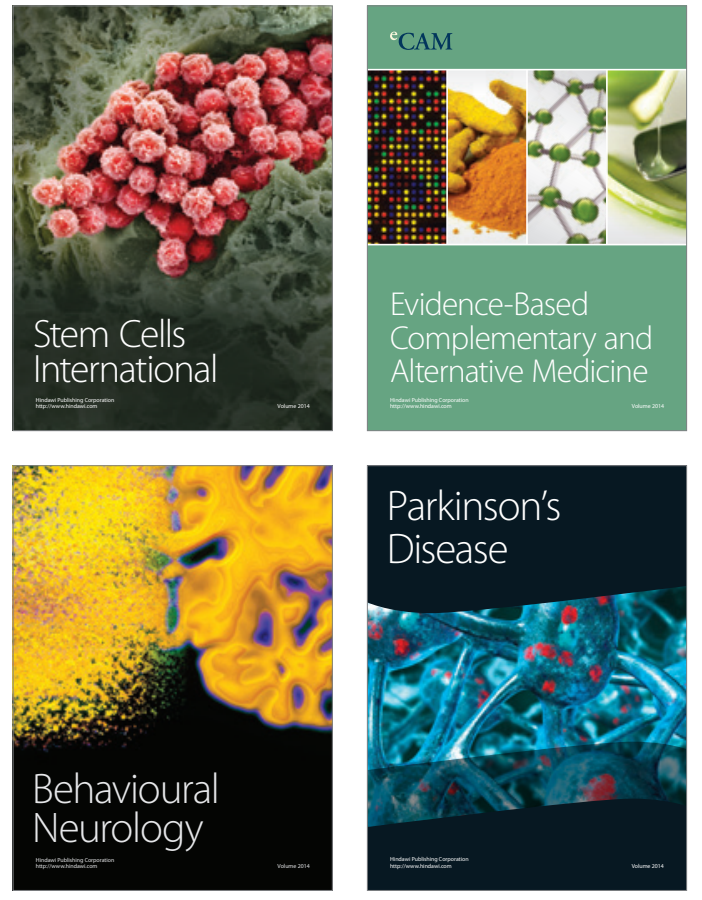
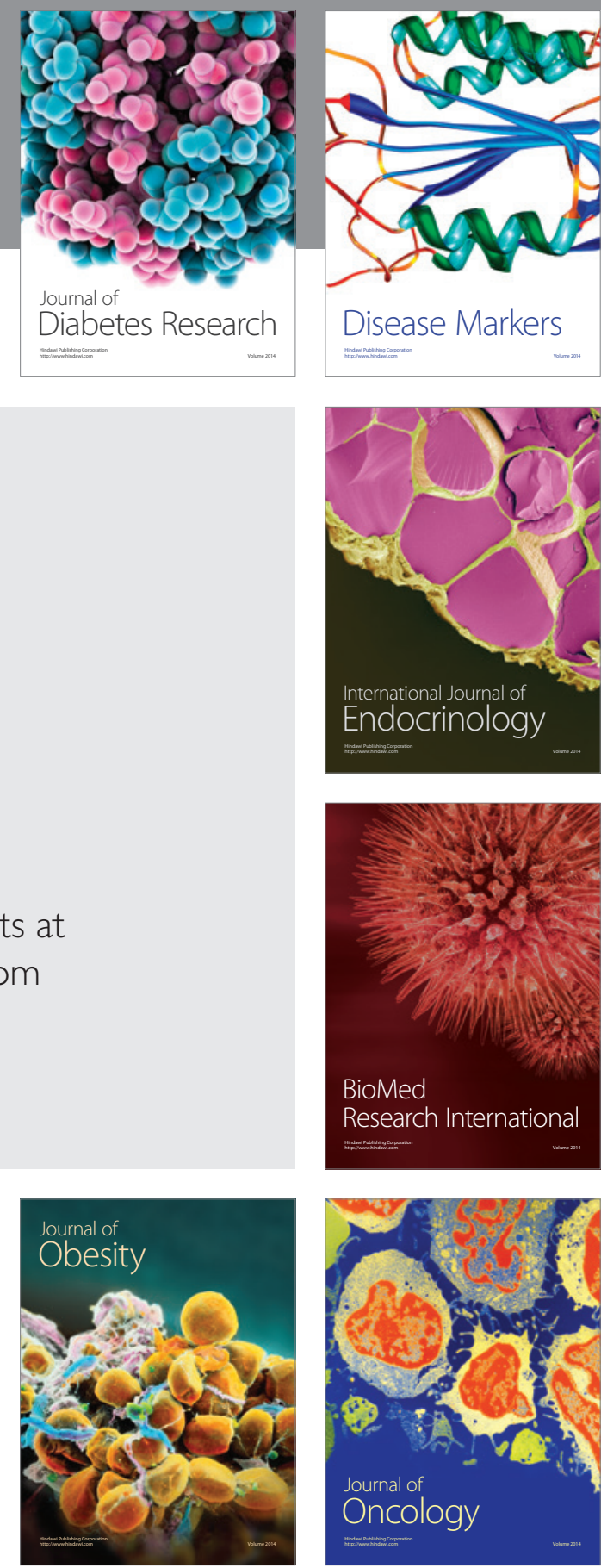

Disease Markers
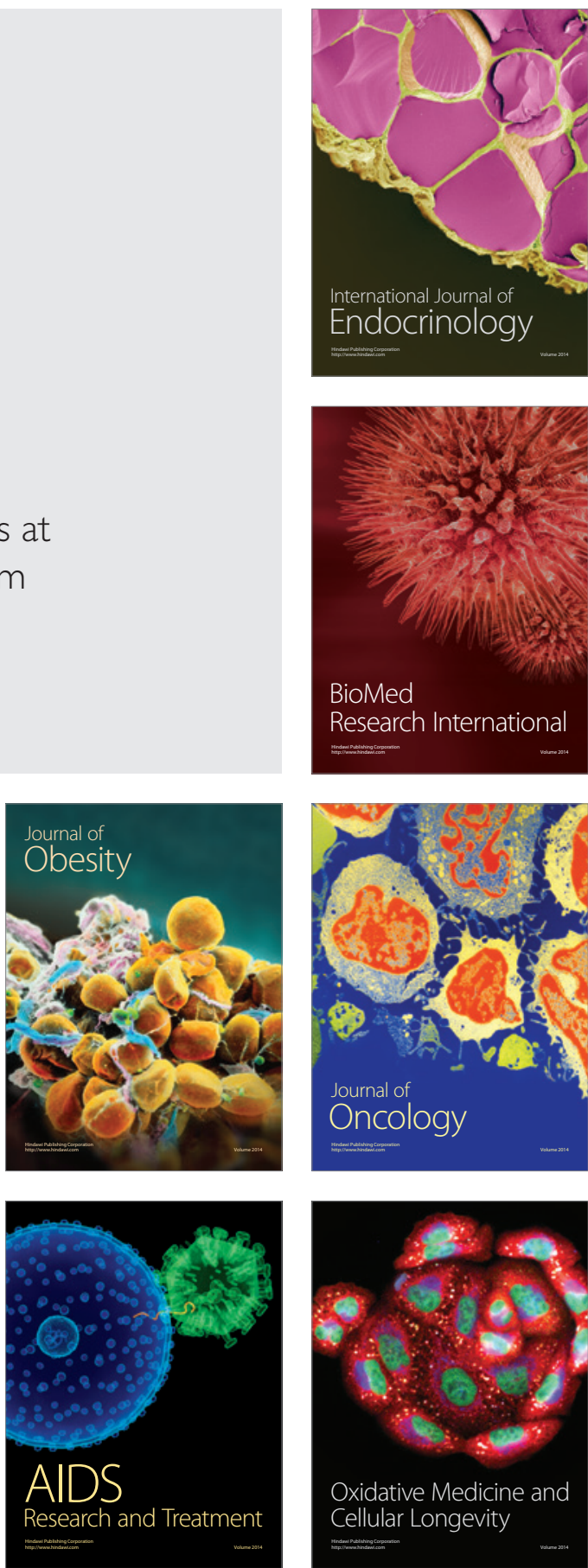